\title{
Effect of nitroso-redox imbalance on male reproduction
}

\author{
Manish Kuchakulla ${ }^{1}$, Thomas Masterson ${ }^{1}$, Himanshu Arora ${ }^{1,2}$, Shathiyah Kulandavelu ${ }^{2}$, Ranjith \\ Ramasamy $^{1,2}$
}

${ }^{1}$ Department of Urology, ${ }^{2}$ The Interdisciplinary Stem Cell Institute, University of Miami, Miller School of Medicine, Miami, FL, USA

Contributions: (I) Conception and design: All authors; (II) Administrative support: R Ramasamy; (III) Provision of study material or patients: None; (IV) Collection and assembly of data: All authors; (V) Data analysis and interpretation: None; (VI) Manuscript writing: All authors; (VII) Final approval of manuscript: All authors.

Correspondence to: Ranjith Ramasamy, MD. 1120 NW 14th Street, Suite 1563, Miami, FL 33136, USA. Email: Ramasamy@miami.edu.

\begin{abstract}
Reactive oxygen species (ROS) and reactive nitrogen species (RNS) are byproducts of normal metabolic processes. They are necessary for normal cellular function and are kept in balance by antioxidant mechanisms. Alterations in levels of ROS and RNS can lead to nitroso-redox imbalance that in turn can negatively affect male reproduction. Strategies to decrease ROS/RNS involve evasion of exposures (smoking, meat intake, pollution, calorie-dense diet), managing lifestyle, and increasing the consumption of antioxidants (vitamin C, vitamin E, alpha-lipoic acid, taurine, quercetin). Targeted therapies focusing on nitroso-redox imbalance can be critical for treatment of male reproductive dysfunction. This review outlines endogenous and exogenous sources of ROS/RNS, adverse effect on male reproduction, and strategies to control nitroso-redox imbalance.
\end{abstract}

Keywords: Nitroso-redox imbalance; reactive oxygen species (ROS); reactive nitrogen species (RNS); nitric oxide synthase (NOS); male reproduction

Submitted Jun 26, 2018. Accepted for publication Jul 30, 2018.

doi: $10.21037 /$ tau.2018.08.14

View this article at: http://dx.doi.org/10.21037/tau.2018.08.14

\section{Introduction}

Reactive oxygen species (ROS) are the byproducts of aerobic metabolism. Hydroxyl radical $\left(\mathrm{OH}^{-}\right)$, superoxide radical $\left(\mathrm{O}_{2}^{-}\right)$, and hydrogen peroxide $\left(\mathrm{H}_{2} \mathrm{O}_{2}\right)$ are some of the isoforms of ROS (1). ROS are kept in balance through the redox environment and the antioxidant system (2). However, at increased levels, ROS lead to tissue damage through oxidative stress by damaging DNA, proteins and lipids. Increased levels of ROS can also trigger apoptosis in cells leading to cell death (3). Oxidative stress is associated with several disease states of increased inflammation like atherosclerosis, asthma, inflammatory bowel disease (IBD), diabetes, Alzheimer's disease, Parkinson's disease, and aging (4-6). Given that ROS have enhanced chemical reactivity, the body has evolved several antioxidant defense mechanisms which considerably delay or inhibit oxidative stress (2). Cellular antioxidant pathways such as dismutase, peroxidase, and catalase enzymes function to neutralize or sequester ROS (2). During times of mildly elevated oxidative stress, tissues are able to respond with additional antioxidant defenses. However, during periods of severe oxidative stress, cell injury and death can occur (7).

In addition to ROS, there is a subset of ROS called reactive nitrogen species (RNS), which are the byproducts of nitric oxide (NO) generation. Peroxynitrite anion $\left(\mathrm{ONOO}^{-}\right)$, nitroxyl ion $\left(\mathrm{NO}^{-}\right)$, nitrosyl-containing compounds, and NO are some of the isoforms of RNS (1). At physiologic levels, RNS and ROS are important for normal cellular functions such as gene activation, blood vessel modification, and enzyme activities. However, at pathologic levels RNS lead to tissue damage and cell death. Similar to ROS, RNS lead to tissue damage through nitrosative stress. Nitrosative stress is a phenomenon in which an imbalance in RNS produces a pathogenic response (8). Similar to oxidative stress, nitrosative stress also arises from dysfunction in the antioxidant defense system. Specifically, elevated levels of 


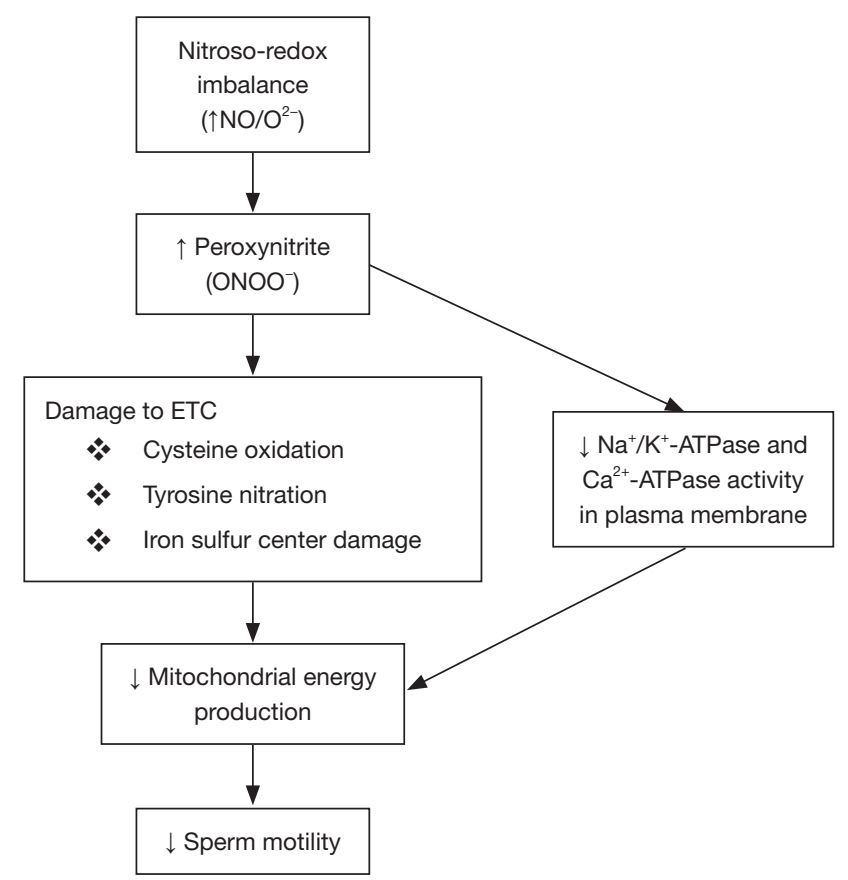

Figure 1 Nitroso-redox imbalance impact on sperm motility. ETC, electron transport chain.

ROS/RNS can damage sperm DNA, lipids, carbohydrates and proteins $(1,9,10)$. Nitroso-redox imbalance can affect male reproduction through its effects on sperm and the hypothalamic-pituitary-gonadal (HPG) axis $(1,9,10)$. In this manuscript, we will review the effect ROS/RNS and their effect on male reproduction.

\section{Synthesis of ROS/RNS}

One of the major sources of ROS comes from the mitochondria due to the numerous electron transfer reactions. ROS are formed by the transformation of oxygen by free electrons whose major source in mitochondria is from cellular respiration [electron transport chain (ETC)] (11). It is estimated that $0.2-2 \%$ of all oxygen consumed is converted to ROS in the ETC (12). These redox reactions occur mainly at complex I and complex III in the ETC which catalyze reactions that directly react with oxygen to generate free radicals (13). Thus, mitochondrial dysfunction that impacts the ETC can lead to excess ROS production (14).

In addition to mitochondria, ROS are produced by other cytoplasmic organelles such as peroxisomes. Xanthine oxidase $(\mathrm{XO})$ is a common source of ROS in the cytoplasm. XO is an important enzyme in the clearance of waste products by converting them to uric acid, however it contributes to high levels of ROS when strongly activated. $\mathrm{XO}$ is also found in peroxisomes which are cellular organelles that produce ROS (15). Peroxisomes' primary role is to break down fatty acids and protein products, which produces hydrogen peroxide and ROS as byproducts. While it's evident that enzymes and organelles are important for normal cellular functions, excessive activity of enzymes such as $\mathrm{XO}$ can lead to oxidative stress.

There are several sources of NO that contribute to nitrosative stress. NO is a commonly found biomolecule that has important roles throughout the body, such as vasodilatation, angiogenesis, inflammation, apoptosis and acts as a neurotransmitter. $\mathrm{NO}$ is able to impact several organ systems because its lipophilic nature allows it to diffuse easily through many cellular components (16). NO is biosynthesized from L-arginine via nitric oxide synthase (NOS). NOS catalyzes the oxidation of the terminal guanidino nitrogen of $\mathrm{L}$-arginine to produce $\mathrm{NO}$ and L-citrulline. Three isoforms of NOS have been identified and these include, neuronal NOS (nNOS, NOS-1), endothelial NOS (eNOS, NOS-3) and inducible NOS (iNOS, NOS-2) (17). While NOS is a major contributor to NO formation, NOS-independent sources have also been identified. It is noted that dietary or exogenous sources of nitrate and nitrite are important contributors to NO production (18). Nitrate is often found in many vegetables and is reduced to nitrite by nitrate reductase which is commonly found in the gastrointestinal tract. Nitrite can be further reduced to $\mathrm{NO}$ by low $\mathrm{pH}$, ascorbic acid, hemoglobin, myoglobin, polyphenols and xanthine oxidoreductase, thus contributing to RNS (19). Taken together, oxidative and nitrosative stress are induced by both cellular organelles and extracellular enzymes.

\section{Effect of oxidative/nitrosative stress on sperm motility}

Oxidative/nitrosative stress can impact sperm motility (Figure 1) by affecting mitochondrial function. Firstly, strong evidence demonstrates nitroso-redox's impacts on sperm motility. Low concentrations of NO enhance motility (20) while medium/high concentrations impair motility (21). The most significant damage from NO occurs when it reacts with superoxide $\left(\mathrm{O}_{2}^{-}\right)$to produce peroxynitrite that can impact cell function and sustainability $(22,23)$. The mechanism by which peroxynitrite can impact 


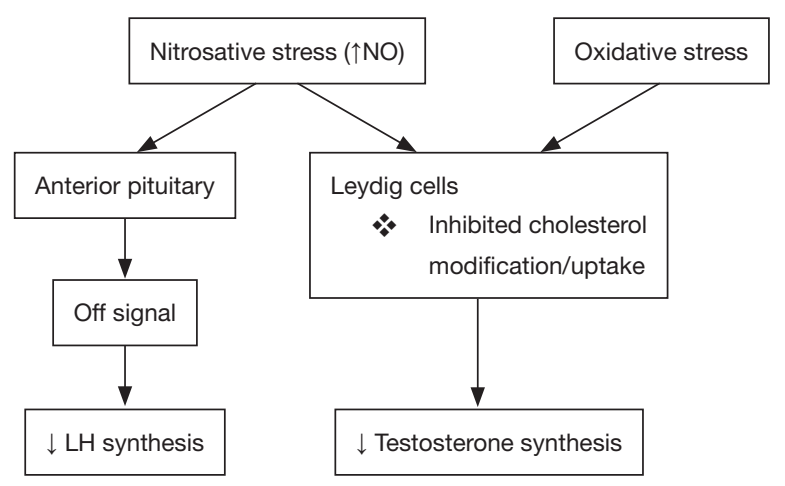

Figure 2 Nitrosative/oxidative stress effect on $\mathrm{LH}$ and testosterone. LH, luteinizing hormone.

sperm motility may involve the inhibition of mitochondrial respiration (17), because peroxynitrite inhibits components of the ETC. Peroxynitrite causes cysteine oxidation, tyrosine nitration and damage to iron sulfur centers (22) that are critical components of the ETC and decreases the mitochondrial membrane potential which is crucial for energy generation. Consequently, energy synthesis is reduced and has a major impact on motility due to the spermatozoa's high metabolic activity and heavy reliance on mitochondrial energy (24). Therefore, sperm motility is reduced by nitroso-redox imbalance's effect on mitochondria.

In addition to nitrosative/oxidative stress' impact on sperm motility through inhibition of the ECT in mitochondria, free radicals also directly impact the sperm plasma membrane and its associated proteins. Peroxynitrite decreases $\mathrm{Na}^{+} / \mathrm{K}^{+}$-ATPase and $\mathrm{Ca}^{2+}$-ATPase activity which are necessary for maintaining osmotic balance, volume, $\mathrm{pH}$, resting membrane potential and for transport of other coupled ions across the membrane $(25,26) . \mathrm{Na}^{+} / \mathrm{K}^{+}$-ATPase plays a primary role in sperm motility. It can be largely found in the mid-piece of the sperm tail and balances the proton gradient in mitochondria (27). Moreover, the $\mathrm{Ca}^{2+}$ ATPase is responsible for regulating sperm motility because it activates the production of cAMP through the regulation of calmodulin, which is an essential factor for motility (28). Thus, inhibited activity in both these enzymes will result in reduced motility. These ATPases are susceptible to nitrosative stress because they have a sulfhydryl (SH)containing enzymes and thiol groups which are depleted by $\mathrm{NO}$ and peroxynitrite (29). Consequently, an imbalance in the nitroso-redox environment can lead to reduced sperm motility by inhibiting these essential membrane proteins.

\section{Nitroso-redox imbalance impairs testosterone and luteinizing hormone (LH) production}

Leydig cells were first described as hormone-producing cells found within the testes in 1850 (30). They play an essential role in testicular development and male fertility by contributing to androgen synthesis and secretion (31). Their function is necessary for proper development, normal masculinization, spermatogenesis and male fertility in general (32). Leydig cells produce more than $95 \%$ of the testosterone in the body and are regulated by $\mathrm{LH}$ production in the anterior pituitary. Leydig cells utilize modified forms of cholesterol in the formation of testosterone. It has been shown that oxidative-nitrosative stress is able to alter mechanisms for steroidogenesis and spermatogenesis (Figure 2).

Nitrosative stress is an inhibitor of Leydig cell steroidogenesis function through NO. Experiments examining the impact of $\mathrm{NO}$ on steroid biosynthesis in Leydig cells demonstrate that $\mathrm{NO}$ administration has an inhibitory effect on steroidogenesis leading to suppressed testosterone levels in mice $(10,33,34)$. Research suggests that NO impacts testosterone synthesis directly by inhibiting cholesterol modification, an important precursor to steroid products (33). However, the hypothalamus-pituitarygonadal axis remains inducible because administration of $\mathrm{GnRH}$ and human chorionic gonadotrophin (hCG) into nitrosatively stressed mice stimulated $\mathrm{LH}$ and testosterone, respectively (10). Thus, nitrosative stress in excess can inhibit testosterone production likely due to hypothalamicpituitary dysfunction.

While NO has demonstrated a strong impact on testosterone levels, it also appears to alter LH secretion. Studies using mice exposed to increased levels NO show decreased activity in the anterior pituitary's ability to secrete LH. It is hypothesized that NO signal to terminate LH release in this region of the brain $(34,35)$. Consequently, decreased LH levels may amplify the negative effects on testosterone production from the testicles because NO decreased the synthesis of testosterone from the Leydig cells directly. It is evident that nitrosative stress is an important factor in steroidogenesis and Leydig cell function.

Oxidative stress is also linked to reduced production of testosterone from Leydig cells (36). Several experiments have demonstrated the impact of oxidative stress on steroidogenesis in Leydig cells and spermatogenesis. Bacterial lipopolysaccharide-induced oxidative stress in mice significantly decreased testosterone synthesis $(37,38)$. 
Table 1 Sources of oxidative/nitrosative stress

\begin{tabular}{lll}
\hline Sources & Effect & Method \\
\hline Smoking & $\uparrow$ ROS/RNS & Tar contains NO \\
& & Induction of free radical release by immune cells \\
Meats & $\uparrow$ ROS/RNS & Nitrates/nitrites used for meat preservation promote NO synthesis \\
& & Heterocyclic amines (HCA) from cooking induce ROS \\
Pollutants & $\uparrow$ ROS/RNS & Radiations, pesticides and xenobiotics damage cell structures \\
& & Alter mitochondrial function, active ROS/RNS producing inflammatory cells, or directly generate free radicals \\
& & Heavy metals can inactive antioxidant reactions \\
& & High fat and carbohydrate diet promote ROS \\
Obesity & $\uparrow$ ROS/RNS & Diminished antioxidant activity \\
\hline
\end{tabular}

ROS, reactive oxygen species; RNS, reactive nitrogen species.

In addition, researchers determined that the expression of a specific protein, steroidogenic acute regulatory protein (StAR), which is essential for cholesterol uptake for testosterone synthesis, was diminished in oxidatively stressed mice (37). It is suggested that a likely mechanism for this dysfunction is through the P450scc enzyme in the mitochondria which catalyzes cholesterol conversion as well as StAR protein expression (38). Accordingly, oxidative stress has indicated a significant inhibitory impact on steroidogenesis in Leydig cells.

\section{Factors influencing oxidative/nitrosative stress}

Exposures such as smoking, meat intake, pollution and obesity can lead to increased oxidative/nitrosative stress (Table 1).

\section{Cigarette smoke promotes free radicals in circulation}

Smoking is associated with an increase in ROS along with numerous adverse health conditions. Specifically, smoking is identified as a promoter of free radicals (4). Cigarette smoke as well as the tar phase contains particularly high levels of NO (39). Many ROS/RNS species are also produced in the lung by neutrophils, alveolar macrophages and eosinophils due to inflammation caused by smoking. It is well established that smokers have a higher number of alveolar macrophages due to chronic inflammation (40). Therefore, NO levels are significantly elevated in smokers when compared to nonsmokers (41). As a result, smoking is a major source of ROS/RNS and contributes to nitroso- redox imbalance.

\section{Meat intake contributes to increased free radicals}

Meat consumption is associated with an increase in free radicals in circulation. One method of inducing RNS is through consuming nitrites directly which have long been used in the preservation of meats. Nitrates/Nitrites offer protection against microbial growth and toxins in processed meats while also promoting the stability of color and freshness which is why they are commonly used $(42,43)$. Nitrite in circulation is normally scavenged and sequestered through reactions with hemoglobin in erythrocytes or by a reaction in the catalase $/ \mathrm{H}_{2} \mathrm{O}_{2}$ system to form nitrate $(44,45)$. However, excess nitrite may react along another pathway and be reduced to $\mathrm{NO}$ by $\mathrm{XO}$, other heme containing enzymes, and ascorbic acid (46-48). Thus, nitrite is able to yield RNS in a pro-oxidative environment which can lead to nitrosative stress (49).

Additionally, meat induces ROS production from byproducts that are formed while cooking them. During the cooking process of meat, heterocyclic amines (HCA) are produced in varying amounts depending on the method and duration. One study suggests that HCA intake contributes to increased oxidative stress independently of other lifestyle factors (50). Therefore, it is suggested that excessive meat consumption can be a source for oxidative stress.

\section{Environmental pollution increases exposure to ROS/RNS}

Over the years, many hazardous chemicals were produced 
Table 2 Strategies to reduce oxidative/nitrosative stress

\begin{tabular}{ll}
\hline Strategy & Effects \\
\hline Lifestyle modification (diet/exercise) & Calorie restriction and healthy diet promote antioxidant activity \\
& Moderate exercise promotes antioxidant status and reduces inflammation \\
Vitamin C (ascorbate) & Has antioxidant function to neutralize hydroxyl, superoxide, and hydrogen peroxide \\
Vitamin E ( $\alpha$-tocopherol) & Inactivates free radicals by donating hydrogen group \\
Alpha-lipoic acid (LA) & Inhibits NO production by NOS \\
& Promotes regenerations of antioxidants such as glutathione and vitamins C and E \\
Taurine & Direct free radical scavenger and antioxidant promoter \\
Quercetin & Antioxidant promoter \\
\hline
\end{tabular}

NOS, nitric oxide synthase.

and released into the environment. These chemicals may adversely impact the health of individuals. Toxic emissions from pollutants found in the air and our water supply such as radiations, pesticides and xenobiotics negatively impact cell structures and organelles. These emissions contain higher levels of molecules such as sulfur dioxide, nitrogen dioxide, carbon monoxide, particle matter and ozone. These molecules produce ROS/RNS either through altering mitochondrial function, activation of ROS/RNS producing inflammatory cells, or by direct generation by the particles themselves (51). Many of these pollutants found their way into the water supply and contribute to heavy metal containing glutathione which alters redox reactions to produce excessive free radicals. These heavy metals also inhibit antioxidant enzymes by binding to and inactivating several functions, leading to weakened free radical protection mechanisms (52). Pollutants drive free radical reactions and promote oxidative/nitrosative stress.

\section{Obesity and diet induce oxidative stress}

Recently, evidence has shown that obesity can induce oxidative stress, leading to male sexual dysfunction (53). Obesity is characterized by an increase in body weight that results in excessive fat accumulation, beyond the limits of physical requirement that is a direct result of physical inactivity (54,55). A strong positive correlation has been demonstrated between BMI and oxidative stress (53). It is believed that conditions commonly associated with obesity such as hyperglycemia, elevated tissue lipid levels, vitamin and mineral deficiencies, chronic inflammation, and impaired mitochondrial function contribute to oxidative stress (56). While ROS production is elevated with obesity, reactive species sequestration is also diminished. Obese individuals have diminished activity in several antioxidant mechanisms such as superoxide dismutase and glutathione peroxidase $(57,58)$. In addition to this, several studies have demonstrated that total antioxidant status is reduced in obese individuals $(53,59,60)$. Therefore, obesityinduced oxidative stress is able to contribute to male sexual dysfunction.

Diet is another contributor to the generation of ROS. A diet high in fat and carbohydrates promotes oxidative stress and inflammation (61). In addition, inadequate consumption of foods rich in antioxidants, such as berries, beans, nuts and broccoli, also contribute to oxidative stress by lessening defense capacities (61). An unbalanced diet will lead to nitroso-redox imbalance and contribute to male sexual dysfunction.

\section{Strategies to decrease oxidative/nitrosative stress}

The negative effects of ROS and RNS on the reproductive system can be accomplished through three strategies: (I) avoidance of elements that increase ROS/RNS (smoking, meat, pollution, fat, inactivity) and (II) improvement of lifestyle, (III) consumption of substances that scavenge ROS/RNS. Methods to avoid substances that increase oxidative stress include controlling smoking, meat intake, alcohol consumption, physical inactivity, and exposure to air pollution (Table 2). Each of these factors has demonstrated significant impacts on free radical levels in the body, thus the primary method of controlling the bodies' oxidative 
environment should be through avoidance of these risk factors. Other strategies to control the oxidative environment are through the consumption of substances that have strong antioxidant potential.

\section{Lifestyle strategies to decrease ROS/RNS}

Dietary management is a robust and important method to combat ROS/RNS production. Diet specifically refers to monitoring the quantity of food consumed as well as the quality of food. Caloric restriction has been shown to enhance the overall antioxidant capacity and aid in maintain optimal intracellular environments (62). However, since long-term calorie restriction is difficult to maintain, several solutions can mimic the health benefits (63). Consumption of compounds such as plant-derived polyphenolic molecules (e.g., quercetin, butein, and piceatannol), insulin-action enhancers (e.g., metformin), or pharmacological agents that inhibit glycolysis (e.g., 2-deoxyglucose) have shown to be promising candidates in mimicking calorie restriction and reducing reactive species production (64). In addition to caloric restriction, consumption of a quality balanced diet reduced ROS production. Vegetables and plant-derived food has a positive impact on maintaining the nitroso-redox balance. Avoiding calorie-dense sugars, saturated fats, and processed foods and replacing them with nutrientdense fruits, vegetables, and legumes is able to strengthen the antioxidant ability and reduce oxidative stress (65). It is important to maintain a well-balanced diet and avoid calorie-dense foods in order to reduce reactive species production (63).

While dietary management is an important component to managing the nitroso-redox environment, exercise also play a major role in ROS/RNS production (63). Physical inactivity is a major cause of obesity and its associated induction of oxidative stress. It is beneficial to maintain consistent physical activity to help prevent the onset of obesity and its associated conditions. Moderate physical activity elevated antioxidant activity, providing protection against oxidation of DNA, proteins and other cellular structures $(66,67)$. Proper maintenance of regular physical activity will be beneficial in reducing ROS/RNS production.

\section{Vitamin C and E}

Vitamins are strong antioxidants and are useful in reducing oxidative/nitrosative stress. One of the strongest and most abundant antioxidants is ascorbate (vitamin C). The body is incapable of synthesizing ascorbate; thus, it must be acquired through diet. Ascorbate reacts with free radicals rendering them stable and unreactive (68). Ascorbate's antioxidant actions are able to neutralize hydroxyl, superoxide, and hydrogen peroxide reactive species (69). Furthermore, $\alpha$-tocopherol (vitamin E) also exhibits strong anti-oxidant potential. Vitamin $\mathrm{E}$ is able to inhibit lipid peroxidation by donating a hydrogen group onto free radicals making them non-reactive (70). Vitamin $\mathrm{E}$ also protects the HPG axis from elevated pituitary and testicular TNF-alpha and IL-6 levels caused by ethanol consumption. Vitamin E's effects suggests it has the ability to be both antiinflammatory and antioxidant (71). Vitamins $\mathrm{C}$ and $\mathrm{E}$ have exhibited strong potential as potent anti-oxidants and can seamlessly be integrated into diets with multivitamins.

\section{Alpha-lipoic acid (LA)}

In addition to vitamins, LA has demonstrated its role as an antioxidant promoter. Diets high in LA, which is found in many vegetables, decrease levels of free radicals in areas of the brain, such as the hypothalamus. LA promotes the regeneration of antioxidants, such as glutathione, Vitamin $\mathrm{E}$ and vitamin $\mathrm{C}$ (72). In addition, another study explored the effect of LA on NO levels in mice. They suggest that LA is able to reduce NO synthesis by inhibiting iNOS. Inhibition of NO directly reduces nitrosative stress (73). Thus, LA promotes the body's natural antioxidant infrastructure to control oxidative stress. Therefore, a LA rich diet is beneficial in reducing ROS/RNS.

\section{Taurine}

Taurine, one of the most abundant free amino acids in mammalian cells, also has antioxidant potential. Taurine reduces oxidative stress by directly scavenging free radicals. Results show that taurine significantly prevented experimentally induced oxidative stress in rats by sodium fluoride $(\mathrm{NaF})$. Taurine also augments antioxidant enzymes activities and glutathione levels in the brain, testes, and epididymis of treated rats. Moreover, taurine reversed NaFinduced elevation in inflammatory biomarkers and caspase-3 activity as well as histological damage in the brain, testes, and epididymis of the oxidatively stressed rats (74). In another study, Zhang et al. induced oxidative stress in men through the use of ergonomic exercises. Subsequently, these subjects were treated with taurine and results demonstrated that taurine attenuated exercise-induced oxidative stress. As a result, oxidative stress induced DNA damage was attenuated (75). 


\section{Quercetin}

Quercetin is a flavonoid found in many fruits and vegetables. Quercetin diminished intracellular oxidative stress caused by exposure to manganese. Manganese exposure suppresses the brain-pituitary-testicular axis function and post-testicular events such as sperm function in rats. Manganese interferes with oxidative phosphorylation in mitochondria leading to the generation of ROS (76). This suppression is likely mediated through persistent oxidative and endocrine disruption caused by manganese in the exposed rats (77). Quercetin is a natural flavonoid that is easily obtained in the diet trough the consumption of fruits and vegetables. When administered with manganese, quercetin significantly $(\mathrm{P}<0.05)$ inhibited manganese-induced elevation in biomarkers of oxidative stress. In addition, quercetin increased antioxidant enzymes activities and glutathione level in the brain, testes and epididymis of the treated rats. It also suppressed inflammatory indices such as caspase- 3 activity which aided in preservation of functioning in the brain, testes, and epididymis in treated rats (78). Quercetin is used as a pharmacological agent in reducing oxidative stress along the brain-pituitary-testicular axis. Thus, quercetin can be a useful mediator of male sexual dysfunction due to nitrosoredox imbalance.

\section{Conclusions}

ROS and RNS are free radicals that in excess lead to oxidative and nitrosative stress. Recent research has implicated ROS/RNS as contributors to infertility and disruption of the HPG axis. Environmental exposures, through diet, contaminants and pollution are sources of ROS/RNS. Strategies to decrease ROS/RNS involve avoidance of exposures and increasing the consumption of antioxidants. At this time, there is limited information from human studies, but animal models conclude that ROS/RNS can impact male reproduction and fertility.

\section{Acknowledgements}

This work was supported by the American Urological Association Research Scholar Award and Stanley Glaser Award to R Ramasamy.

\section{Footnote}

Conflicts of Interest: The authors have no conflicts of interest to declare.

\section{References}

1. Doshi SB, Khullar K, Sharma RK, et al. Role of reactive nitrogen species in male infertility. Reprod Biol Endocrinol 2012;10:109.

2. Gutteridge JM. Lipid peroxidation and antioxidants as biomarkers of tissue damage. Clin Chem 1995;41:1819-28.

3. Ghosh P, Mukherjee S, Bhoumik A, et al. A novel epididymal quiescence factor inhibits sperm motility by modulating NOS activity and intracellular NO-cGMP pathway. J Cell Physiol 2018;233:4345-59.

4. Pandey R, Singh M, Singhal U, et al. Oxidative/Nitrosative stress and the pathobiology of chronic obstructive pulmonary disease. J Clin Diagn Res 2013;7:580-8.

5. Pandey R, Gupta S, Lal H, et al. Hyperhomocysteinemia and cardiovascular disease: The nutritional perspectives. Indian J Clin Biochem 2000;15:20-30.

6. Eiserich JP, van der Vliet A, Handelman GJ, et al. Dietary antioxidants and cigarette smoke-induced biomolecular damage: a complex interaction. Am J Clin Nutr 1995;62:1490S-1500S.

7. Betteridge DJ. What is oxidative stress? Metabolism 2000;49:3-8.

8. Gawda A, Majka G, Nowak B, et al. Air pollution, oxidative stress, and exacerbation of autoimmune diseases. Cent Eur J Immunol 2017;42:305-12.

9. Vieira NMG, Losano JDA, Angrimani DSR, et al. Induced sperm oxidative stress in dogs: Susceptibility against different reactive oxygen species and protective role of seminal plasma. Theriogenology 2018;108:39-45.

10. Masterson TA, Arora H, Kulandavelu S, et al. S-Nitrosoglutathione Reductase (GSNOR) Deficiency Results in Secondary Hypogonadism. J Sex Med 2018;15:654-61.

11. Alfadda AA, Sallam RM. Reactive oxygen species in health and disease. J Biomed Biotechnol 2012;2012:936486.

12. Balaban RS, Nemoto S, Finkel T. Mitochondria, oxidants, and aging. Cell 2005;120:483-95.

13. Brieger K, Schiavone S, Miller FJ Jr, et al. Reactive oxygen species: from health to disease. Swiss Med Wkly 2012;142:w13659.

14. McMurray F, Patten DA, Harper ME. Reactive Oxygen Species and Oxidative Stress in Obesity-Recent Findings and Empirical Approaches. Obesity (Silver Spring) 2016;24:2301-10.

15. Di Meo S, Reed TT, Venditti P, et al. Role of ROS and RNS Sources in Physiological and Pathological Conditions. Oxid Med Cell Longev 2016;2016:1245049. 
16. Archer S. Measurement of nitric oxide in biological models. FASEB J 1993;7:349-60.

17. Rosselli M, Keller PJ, Dubey RK. Role of nitric oxide in the biology, physiology and pathophysiology of reproduction. Hum Reprod Update 1998;4:3-24.

18. Lundberg JO, Weitzberg E, Gladwin MT. The nitratenitrite-nitric oxide pathway in physiology and therapeutics. Nat Rev Drug Discov 2008;7:156-67.

19. Moncada S, Palmer RM, Higgs EA. Nitric oxide: physiology, pathophysiology, and pharmacology. Pharmacol Rev 1991;43:109-42.

20. Hellstrom WJ, Bell M, Wang R, et al. Effect of sodium nitroprusside on sperm motility, viability, and lipid peroxidation. Fertil Steril 1994;61:1117-22.

21. Rosselli M, Dubey RK, Imthurn B, et al. Effects of nitric oxide on human spermatozoa: evidence that nitric oxide decreases sperm motility and induces sperm toxicity. Hum Reprod 1995;10:1786-90.

22. Pacher P, Beckman JS, Liaudet L. Nitric oxide and peroxynitrite in health and disease. Physiol Rev 2007;87:315-424.

23. Uribe P, Boguen R, Treulen F, et al. Peroxynitritemediated nitrosative stress decreases motility and mitochondrial membrane potential in human spermatozoa. Mol Hum Reprod 2015;21:237-43.

24. Kisa U, Basar MM, Ferhat M, et al. Testicular tissue nitric oxide and thiobarbituric acid reactive substance levels: evaluation with respect to the pathogenesis of varicocele. Urol Res 2004;32:196-9.

25. Skou JC, Esmann M. The Na,K-ATPase. J Bioenerg Biomembr 1992;24:249-61.

26. Vignini A, Nanetti L, Buldreghini E, et al. The production of peroxynitrite by human spermatozoa may affect sperm motility through the formation of protein nitrotyrosine. Fertil Steril 2006;85:947-53.

27. Sanchez G, Nguyen AN, Timmerberg B, et al. The $\mathrm{Na}, \mathrm{K}$-ATPase alpha4 isoform from humans has distinct enzymatic properties and is important for sperm motility. Mol Hum Reprod 2006;12:565-76.

28. Boni R, Gualtieri R, Talevi R, et al. Calcium and other ion dynamics during gamete maturation and fertilization. Theriogenology 2007;68 Suppl 1:S156-64.

29. Vignini A, Buldreghini E, Nanetti L, et al. Free thiols in human spermatozoa: are $\mathrm{Na}+\mathrm{K}+$-ATPase, Ca2+-ATPase activities involved in sperm motility through peroxynitrite formation? Reprod Biomed Online 2009;18:132-40.

30. Wainwright EN, Wilhelm D. The game plan: cellular and molecular mechanisms of mammalian testis development.
Curr Top Dev Biol 2010;90:231-62.

31. Tremblay JJ. Molecular regulation of steroidogenesis in endocrine Leydig cells. Steroids 2015;103:3-10.

32. Hu W, Shi L, Li MY, et al. Adrenomedullin protects Leydig cells against lipopolysaccharide-induced oxidative stress and inflammatory reaction via MAPK/NF-kappaB signalling pathways. Sci Rep 2017;7:16479.

33. Del Punta K, Charreau EH, Pignataro OP. Nitric oxide inhibits Leydig cell steroidogenesis. Endocrinology 1996;137:5337-43.

34. Adams ML, Meyer ER, Sewing BN, et al. Effects of nitric oxide-related agents on rat testicular function. J Pharmacol Exp Ther 1994;269:230-7.

35. Ceccatelli S, Hulting AL, Zhang X, et al. Nitric oxide synthase in the rat anterior pituitary gland and the role of nitric oxide in regulation of luteinizing hormone secretion. Proc Natl Acad Sci U S A 1993;90:11292-6.

36. Wang JY, Lee YJ, Chou MC, et al. Astaxanthin protects steroidogenesis from hydrogen peroxide-induced oxidative stress in mouse Leydig cells. Mar Drugs 2015;13:1375-88.

37. Reddy MM, Mahipal SV, Subhashini J, et al. Bacterial lipopolysaccharide-induced oxidative stress in the impairment of steroidogenesis and spermatogenesis in rats. Reprod Toxicol 2006;22:493-500.

38. Tsai SC, Lu CC, Lin CS, et al. Antisteroidogenic actions of hydrogen peroxide on rat Leydig cells. J Cell Biochem 2003;90:1276-86.

39. Jindal SK, Aggarwal AN, Chaudhry K, et al. A multicentric study on epidemiology of chronic obstructive pulmonary disease and its relationship with tobacco smoking and environmental tobacco smoke exposure. Indian J Chest Dis Allied Sci 2006;48:23-9.

40. Rahman I, MacNee W. Oxidant/antioxidant imbalance in smokers and chronic obstructive pulmonary disease. Thorax 1996;51:348-50.

41. Wadhwa D, Bey A, Hasija M, et al. Determination of levels of nitric oxide in smoker and nonsmoker patients with chronic periodontitis. J Periodontal Implant Sci 2013;43:215-20.

42. Skibsted LH. Nitric oxide and quality and safety of muscle based foods. Nitric Oxide 2011;24:176-83.

43. Honikel KO. The use and control of nitrate and nitrite for the processing of meat products. Meat Sci 2008;78:68-76.

44. Doyle MP, Herman JG, Dykstra RL. Autocatalytic oxidation of hemoglobin induced by nitrite: activation and chemical inhibition. J Free Radic Biol Med 1985;1:145-53.

45. Herold S, Rehmann FJ. Kinetics of the reactions of nitrogen monoxide and nitrite with ferryl hemoglobin. 
Free Radic Biol Med 2003;34:531-45.

46. Zhang Z, Naughton D, Winyard PG, et al. Generation of nitric oxide by a nitrite reductase activity of xanthine oxidase: a potential pathway for nitric oxide formation in the absence of nitric oxide synthase activity. Biochem Biophys Res Commun 1998;249:767-72.

47. Millar TM, Stevens CR, Benjamin N, et al. Xanthine oxidoreductase catalyses the reduction of nitrates and nitrite to nitric oxide under hypoxic conditions. FEBS Lett 1998;427:225-8.

48. Godber BL, Doel JJ, Sapkota GP, et al. Reduction of nitrite to nitric oxide catalyzed by xanthine oxidoreductase. J Biol Chem 2000;275:7757-63.

49. Villaverde A, Parra V, Estevez M. Oxidative and nitrosative stress induced in myofibrillar proteins by a hydroxylradical-generating system: impact of nitrite and ascorbate. J Agric Food Chem 2014;62:2158-64.

50. Carvalho AM, Miranda AM, Santos FA, et al. High intake of heterocyclic amines from meat is associated with oxidative stress. Br J Nutr 2015;113:1301-7.

51. Risom L, Moller P, Loft S. Oxidative stress-induced DNA damage by particulate air pollution. Mutat Res 2005;592:119-37.

52. Kaur R, Kaur J, Mahajan J, et al. Oxidative stress-implications, source and its prevention. Environ Sci Pollut Res Int 2014;21:1599-613.

53. Vincent HK, Taylor AG. Biomarkers and potential mechanisms of obesity-induced oxidant stress in humans. Int J Obes (Lond) 2006;30:400-18.

54. Sikaris KA. The clinical biochemistry of obesity. Clin Biochem Rev 2004;25:165-81.

55. Laskowski ER. The role of exercise in the treatment of obesity. PM R 2012;4:840-4; quiz 844.

56. Manna P, Jain SK. Obesity, Oxidative Stress, Adipose Tissue Dysfunction, and the Associated Health Risks: Causes and Therapeutic Strategies. Metab Syndr Relat Disord 2015;13:423-44.

57. Olusi SO. Obesity is an independent risk factor for plasma lipid peroxidation and depletion of erythrocyte cytoprotectic enzymes in humans. Int J Obes Relat Metab Disord 2002;26:1159-64.

58. Ozata M, Mergen M, Oktenli C, et al. Increased oxidative stress and hypozincemia in male obesity. Clin Biochem 2002;35:627-31.

59. Vincent HK, Morgan JW, Vincent KR. Obesity exacerbates oxidative stress levels after acute exercise. Med Sci Sports Exerc 2004;36:772-9.

60. Fenkci V, Fenkci S, Yilmazer M, et al. Decreased total antioxidant status and increased oxidative stress in women with polycystic ovary syndrome may contribute to the risk of cardiovascular disease. Fertil Steril 2003;80:123-7.

61. Patel C, Ghanim H, Ravishankar S, et al. Prolonged reactive oxygen species generation and nuclear factorkappaB activation after a high-fat, high-carbohydrate meal in the obese. J Clin Endocrinol Metab 2007;92:4476-9.

62. Lee DW, Yu BP. Modulation of free radicals and superoxide dismutases by age and dietary restriction. Aging (Milano) 1990;2:357-62.

63. Poljsak B. Strategies for reducing or preventing the generation of oxidative stress. Oxid Med Cell Longev 2011;2011:194586.

64. Ingram DK, Zhu M, Mamczarz J, et al. Calorie restriction mimetics: an emerging research field. Aging Cell 2006;5:97-108.

65. Arguelles S, Gomez A, Machado A, et al. A preliminary analysis of within-subject variation in human serum oxidative stress parameters as a function of time. Rejuvenation Res 2007;10:621-36.

66. Radak Z, Pucsuk J, Boros S, et al. Changes in urine 8-hydroxydeoxyguanosine levels of super-marathon runners during a four-day race period. Life Sci 2000;66:1763-7.

67. Vasankari TJ, Kujala UM, Vasankari TM, et al. Effects of acute prolonged exercise on-serum and LDL oxidation and antioxidant defences. Free Radic Biol Med 1997;22:509-13.

68. Rinnerthaler M, Bischof J, Streubel MK, et al. Oxidative stress in aging human skin. Biomolecules 2015;5:545-89.

69. Buettner GR. The pecking order of free radicals and antioxidants: lipid peroxidation, alpha-tocopherol, and ascorbate. Arch Biochem Biophys 1993;300:535-43.

70. Jiang Q. Natural forms of vitamin E: metabolism, antioxidant, and anti-inflammatory activities and their role in disease prevention and therapy. Free Radic Biol Med 2014;72:76-90.

71. Zhu Q, Emanuele MA, LaPaglia N, et al. Vitamin E prevents ethanol-induced inflammatory, hormonal, and cytotoxic changes in reproductive tissues. Endocrine 2007;32:59-68.

72. Veskovic M, Mladenovic D, Jorgacevic B, et al. Alphalipoic acid affects the oxidative stress in various brain structures in mice with methionine and choline deficiency. Exp Biol Med (Maywood) 2015;240:418-25.

73. Liang JF, Akaike T. Inhibition of nitric oxide synthesis in primary cultured mouse hepatocytes by alpha-lipoic acid. Chem Biol Interact 2000;124:53-60.

74. Adedara IA, Olabiyi BF, Ojuade TD, et al. Taurine reverses 
sodium fluoride-mediated increase in inflammation, caspase- 3 activity, and oxidative damage along the brain-pituitary-gonadal axis in male rats. Can J Physiol Pharmacol 2017;95:1019-29.

75. Zhang M, Izumi I, Kagamimori S, et al. Role of taurine supplementation to prevent exercise-induced oxidative stress in healthy young men. Amino Acids 2004;26:203-7.

76. Gunter KK, Aschner M, Miller LM, et al. Determining the oxidation states of manganese in NT2 cells and

Cite this article as: Kuchakulla M, Masterson T, Arora H, Kulandavelu S, Ramasamy R. Effect of nitroso-redox imbalance on male reproduction. Transl Androl Urol 2018;7(6):968-977. doi: 10.21037/tau.2018.08.14 cultured astrocytes. Neurobiol Aging 2006;27:1816-26.

77. Adedara IA, Abolaji AO, Awogbindin IO, et al. Suppression of the brain-pituitary-testicular axis function following acute arsenic and manganese co-exposure and withdrawal in rats. J Trace Elem Med Biol 2017;39:21-9.

78. Adedara IA, Subair TI, Ego VC, et al. Chemoprotective role of quercetin in manganese-induced toxicity along the brain-pituitary-testicular axis in rats. Chem Biol Interact 2017;263:88-98. 